\title{
Validade da escala de depressão: relação com ansiedade e stress laboral
}

\author{
Validity of the depression scale related to \\ anxiety and occupational stress
}

\author{
Makilim Nunes BAPTISTA \\ Adriana Munhoz CARNEIRO²
}

\begin{abstract}
Resumo
Considerando os dados estatísticos que indicam a depressão como um dos transtornos que mais acometem as pessoas na atualidade e a carência de instrumentos construídos no Brasil sobre o tema, este estudo teve como objetivo buscar evidências de validade para um conjunto de indicadores, inicialmente denominado Escala de Depressão, baseado na relação com outras variáveis, no caso, o Inventário de Ansiedade de Beck e a Escala de Vulnerabilidade ao Estresse no Trabalho. Participaram desta pesquisa 121 estudantes universitários do interior do Estado de São Paulo que exerciam alguma função laboral. Os participantes responderam coletivamente a um questionário de identificação juntamente com a Escala de Depressão, o Inventário de Ansiedade de Beck e a Escala de Vulnerabilidade ao Estresse no Trabalho. Os resultados apontaram correlações positivas entre os sintomas depressivos com ansiedade e stress laboral, o que vem ao encontro da literatura nacional e internacional.
\end{abstract}

Unitermos: Ansiedade. Avaliação psicológica. Depressão. Stress laboral. Validade.

\begin{abstract}
By considering statistical data that show depression to be one of the mental disorders that currently affects most people and the lack of psychometric tests formulated in Brazil in respect of this subject, the objective of this study was to find evidence of validity for a set of indicators, initially designated as the Depression Scale, based on the relationship with other variables, using the Beck Anxiety Inventory and the Occupational Stress Vulnerability Scale. A total of 121 undergraduates from the interior of the state of São Paulo, who have some kind of job, took part in this study, responding collectively to these instruments and a questionnaire for identification. Results showed positive correlations between depression symptoms, occupational stress and anxiety, as both national and international literature demonstrates.
\end{abstract}

Uniterms: Anxiety. Psychological assessment. Depression. Occupational stress. Validity.

O humor deprimido, quando presente de forma frequente, intensa e duradoura, pode ser considerado patológico, levando a grande sofrimento por parte de quem o experimenta, pela sensação de não conseguir controlar os sentimentos e pensamentos (Fester, 1983; Sadock \& Sadock, 2007). Há mais de 2000 anos, o humor

$\boldsymbol{\nabla \nabla \nabla \nabla}$

1 Universidade São Francisco, Programa de Pós Graduação Stricto Sensu em Psicologia. Av. Alexandre Rodrigues Barbosa, 45, Centro, 13251-900, Itatiba, SP, Brasil. Correspondência para/Correspondence to: M.N. BAPTISTA. E-mail: <makilim.baptistasãofrancisco.com.br>.

2 Universidade São Francisco, Curso de Psicologia. Itatiba, SP, Brasil. 
deprimido vem sendo estudado, com diversas outras nomenclaturas. Uma das primeiras classificações que se têm notícia foi feita por Hipócrates, que definia o humor deprimido como mela cholis (melancolia, bile negra) e indicava um desequilíbrio das emoções. É importante levar em consideração que o diagnóstico, a doença, seu nome e uso são relativos à cultura em que estão inseridos; assim sendo, conceitos de tristeza, melancolia e depressão tinham variação cultural, conforme seu papel social e o período histórico. Para Aristóteles, por exemplo, a melancolia era indicadora de pessoas com maior inteligência, com maior inspiração, e surgia mediante a ingestão de derivados do leite. Para a igreja católica, na Idade Média, a melancolia já significava o afastamento de Deus e era considerada um pecado capital. Assim, ao longo dos séculos, ora foi considerada como positiva, ora como negativa (Cordás, 1997; Mead, s.d.; Wong, 2007).

A partir da tradução do termo melancolia para o latim, passou-se a utilizar a palavra depressão (depremere), sendo consagrada por Emil Kraepelin - conhecido por organizar a nosografia psiquiátrica, em 1899, na sexta edição de seu compêndio -, que associou a depressão à mania, sendo a melancolia classificada como um de seus sintomas. O termo depressão foi introduzido somente na segunda edição do Diagnostical and Statistical Manual of Mental Disorders (DSM-II), em 1968, como depressão neurótica, e, atualmente, ela é considerada um transtorno independente, nominada dentro das categorias de humor ou de afeto (Cordás, 1997; Sadock \& Sadock, 2007; Wong, 2007).

O transtorno depressivo maior, uma das denominações atuais, é considerado de etiologia multifatorial, uma vez que possui aspectos genéticos, neuroquímicos e sociais, e acomete todas as idades. As estatísticas indicam que é o transtorno mental que mais afeta a população mundial, e que será a doença mais comum em 2020 (World Health Organization - WHO, 2006). A probabilidade de um indivíduo desenvolver o quadro depressivo ao longo da vida varia de 10\% a 25\% em mulheres e de $5 \%$ a $12 \%$ nos homens, sendo mais comum em indivíduos entre 20 e 40 anos de idade (American Psychological Association - APA, 2002; WHO, 2006).

A depressão interfere de forma significativa na vida social do indivíduo e em sua qualidade de vida, 346 podendo muitas vezes afetar a produtividade laboral
(Pearlin, 1982). Sua relação com outras variáveis, tais como ansiedade e stress laboral, vem sendo estudada com mais ênfase, principalmente devido à importância dos problemas de saúde e de trabalho na vida do indivíduo; assim, baixos níveis de segurança, de autoridade e de poder de decisão laborais encontram-se associados com aumento de depressão (Sparrenberger, Santos \& Lima, 2004, Schnittker, 2010).

Todo ser humano possui um limiar de stress, que quando reduzido, torna o indivíduo mais vulnerável, aumentando as chances de desenvolver sintomas de stress clínicos (Guic, Bilbao \& Bertin, 2002). Assim, a forma com que a pessoa interpretará a situação como ameaçadora é que a deixará vulnerável e não a situação em si; por isso, as pessoas não são afetadas da mesma maneira pelo mesmo evento estressor (Beck, Rush, Shaw \& Emery, 1982; Lazarus, 1976; Pearlin, 1982). Grote, Bledsoe, Larkin, Lemay e Brown (2007) verificaram que, independentemente da raça ou da classe social, mulheres com pensamentos pessimistas acerca do futuro tinham tendências a ter mais stress e sintomas depressivos, e que quanto maior o nível de estressores crônicos, maior a vulnerabilidade das participantes aos sintomas depressivos.

Alguns estudos de revisão indicam ainda que os fatores genéticos podem influenciar na vulnerabilidade ao stress e no desenvolvimento de depressão. Warner, Wickramaratne e Weissman (2008) e Schittker (2010) enfatizam que estudos realizados com gêmeos apontam para resultados que indicam que, apesar da predisposição genética, ela por si só não explica o desenvolvimento da depressão ou do stress. Os autores abordam as estreitas ligações entre stress e depressão, considerando que ambos envolvem a ativação dos mesmos neurotransmissores. Em uma perspectiva biológica, nota-se que os neurotransmissores que se relacionam com o desenvolvimento da depressão, tais como a serotonina e o GABA, também influenciam o desenvolvimento da ansiedade, verificando-se que esses construtos parecem demonstrar coocorrência de sintomas e, consequentemente, merecem grande atenção no processo de avaliação psicológica (Morais, Baptista, Calais \& Inocente, 2004; Warner et al., 2008).

Conforme Becketal. (1982) e Keenan, Feng, Hipwell e Klostermann (2009), as relações entre ansiedade e depressão não são tão distantes, pois os pacientes de- 
pressivos geralmente relatam um aumento nos sintomas de ansiedade quando a depressão diminui. Segundo a Anxiety Disorders Association of America - ADAA (ADAA, 2009), metade dos pacientes diagnosticados com depressão também são diagnosticados com ansiedade, e, apesar de serem transtornos clinicamente diferentes, as pessoas podem apresentar sintomas de ansiedade e de depressão conjuntamente, tais como nervosismo, irritabilidade e problemas de concentração.

Os transtornos de ansiedade, de acordo com a Anxiety Disorders Association of America (ADAA, 2009), acometem aproximadamente 40 milhões de americanos adultos. A ansiedade caracteriza-se por uma ameaça interna desconhecida, que envolve sentimentos de apreensão, alerta, tensão e desconforto perante a antecipação de um evento que ainda não ocorreu, sendo por vezes acompanhada por sintomas autonômicos, como palpitações e inquietação (Sadock \& Sadock, 2007). Como na depressão, as mulheres, influenciadas por fatores genéticos e psicossociais, apresentam risco maior de desenvolver transtornos de ansiedade (ADAA, 2009; Sadock \& Sadock, 2007).

Um ponto a ser considerado na avaliação desses fenômenos é que níveis elevados de stress ou de ansiedade podem estar associados a muitos dos sintomas físicos - dores de cabeça, diminuição de libido, insônia e fadiga - quanto mentais - redução da capacidade de concentração e preocupação excessiva - também desencadeados pela depressão (Pearlin, 1982; Lazarus, 1983).

Fatores como mudanças de chefia, cargo, condição financeira, estratégias inadequadas de enfrentamento, dificuldades para lidar com o superior, horários de trabalho ininterruptos, por exemplo, têm sido apontados como fatores de vulnerabilidade para o desenvolvimento de stress laboral, ansiedade e depressão (Corrêa, 2008; Guic et al., 2002; Oswaldo, 2009).

Palmeira (2009) realizou um estudo para buscar evidências de validade para a Escala de Depressão (EDEP) com a Escala de Vulnerabilidade ao Stress no Trabalho (EVENT), em uma amostra de 301 policiais do Estado de São Paulo. Os resultados indicaram correlações da EDEP com a EVENT no Fator Clima e Funcionamento Organizacional $(r=0,31)$, Pressão no Trabalho $(r=0,25)$, Infraestrutura e Rotina $(r=0,28)$ e no Fator Total $(r=0,34)$. As mulheres pontuaram mais na EDEP e na Infraestrutura e Rotina, o que demonstra que, apesar de correlações baixas, o stress laboral correlacionou-se positivamente com os sintomas depressivos, indicando relação entre os construtos.

Oswaldo (2009), em um estudo para buscar evidências de validade de critério para a EVENT, realizou um estudo com uma amostra de 185 gestores das áreas educacional e de saúde, com idade média de 39 anos (Desvio-Padrão-DP=9,96), sendo 60\% do sexo feminino. Os instrumentos aplicados foram a EDEP, o Inventário de Sintomas de Stress para Adultos de Lipp (ISSL), WHOQOL-Brefe a Escala de Coping Ocupacional (ECO). Os resultados indicaram que quanto maior a vulnerabilidade ao stress no trabalho, maior o stress mensurado pelo ISSL $(r=0,42)$ e a sintomatologia depressiva $(r=0,39)$.

Um estudo realizado por Isyanov e Calamari (2004) avaliou se o stress podia mediar relações entre sintomas de ansiedade e de depressão. Participaram do estudo 85 adolescentes de ambos os sexos, provenientes de cursos de graduação. Os resultados apontaram que a percepção do stress foi mediadora da relação entre ansiedade e depressão. Verificou-se que a ansiedade esteve relacionada à sintomatologia depressiva e ao stress laboral, como consequência dessas rápidas mudanças em que o tempo torna-se escasso e as atividades devem ser realizadas de forma rápida e eficaz.

Visando validar um questionário de depressão traço-estado na Colômbia, Otálvaro (2007) realizou uma pesquisa com dois grupos: o primeiro composto por 300 universitários, sendo 137 do sexo masculino; e o segundo, por 300 adolescentes, sendo 136 do sexo masculino, com idade média de 15,15 (DP=1,21). Os participantes responderam ao Questionário de Depressão Traço-Estado - ST/DEP, ao BDI, Questionário Básico de Depressão - CBD, Inventário de Ansiedade Traço-Estado - STAI e ao Inventário de Expressão de Ira Traço-Estado - STAXI-2. Os resultados evidenciaram correlações significativas entre os instrumentos de depressão e o de ansiedade traço-estado, de forma que as correlações encontradas entre traço e/ou estado de depressão e ansiedade variaram de 0,46 a 0,74. Os resultados apresentaram-se como indicativos da comorbidade e da sobreposição entre os sintomas de ansiedade e de depressão.

Apesar da estreita relação entre os construtos, ainda se carece de pesquisas que abordem o tema, o 
que indica a necessidade de se ampliarem os estudos no País. Para isso, é importante levar em consideração que a maioria dos instrumentos relatados nas pesquisas foi construída e desenvolvida fora do Brasil e, portanto, necessita, por exemplo, ser adaptada a fatores culturais, pois a criação e a validação de instrumentos que consideram a cultura têm se tornado um dos objetivos de psicometristas e psicólogos (Crombach, 1996; Pasquali, 1999; Pereira \& Amaral, 2004).

Tal como Otálvaro (2007) afirma, a utilização de instrumentos criados e validados em outra cultura diminui consideravelmente os níveis de validade e de confiabilidade dos testes; assim, estudos sobre parâmetros psicométricos para criação de instrumentos são de relevante importância, principalmente para verificar se eles são válidos e confiáveis (Crombach, 1996; Pasquali, 1999). O conceito fundamental para se avaliar um instrumento é a validade, que se refere à interpretabilidade dos dados. Portanto, é a validade de um instrumento psicológico que permitirá inferir os escores obtidos pelo teste (Crombach, 1996; Urbina, 2007).

Desse modo, o objetivo principal desta pesquisa foi buscar evidências de validade relacionadas com outras variáveis para a EDEP, avaliando, para isso, relações entre medidas de ansiedade e stress laboral em universitários. Esse tipo de evidência tem como função verificar o grau de associação entre construtos relacionados (American Educational Research Association AERA, American Psychological Association - APA e Nacional Council on Measurement in Education NCME,1999).

\section{Método}

\section{Participantes}

\section{A amostra do presente estudo foi composta por} 121 universitários do interior do Estado de São Paulo,

Quadro 1. Itens da escala de depressão. dos cursos de direito ( $n=79)$ e enfermagem ( $n=42)$, que exerciam alguma função laboral. Do total amostral, 69 eram mulheres; a idade dos participantes variou de 17 a 55 anos, com média de 26,38 (DP=7,57).

\section{Instrumentos}

Escala de Depressão (EDEP) (Baptista, 2009): esta escala foi idealizada com base no DSM-IV-TR (APA, 2002), CID-10 (Organização Mundial da Saúde - OMS, 1993), teoria cognitiva (Beck et al., 1982) e terapia comportamental da depressão (Ferster, 1983). Trata-se de um conjunto de 75 itens, baseado em 26 indicadores - tais como desesperança, choro, tristeza, hipocondria, falta de libido, possuindo frases que apontam extremos (frases negativas e frases positivas), para que o sujeito indique em uma variação de cinco pontos como se sente em relação àquele item. A pontuação varia de 0 a 300, aumentando a pontuação de acordo com os sintomas. O presente estudo ainda é preliminar, não havendo um ponto de corte definido para o instrumento por ainda não haver uma amostra clínica suficiente para as análises normativas, apesar de Souza (2010) já ter encontrado 0,97 de precisão, sensibilidade e especificidade acima de 0,80 em uma amostra conjunta (clínica e não clínica) de 771 participantes. Apresentam-se, abaixo, exemplos dos itens no Quadro 1.

Escala de Vulnerabilidade ao Stress no Trabalho (EVENT) (Sisto, Baptista, Noronha \& Santos, 2007): é uma escala tipo Likert de três pontos, que avalia estressores laborais, com pontuação variando de 0 a 80. A escala, após análise fatorial dos componentes principais com rotação varimax, ficou composta por três dimensões (variância explicada de 37,44\%). A análise por Teoria de Resposta ao Item (TRI) demonstrou que todos os itens apresentaram índices de Infit ajustados ao modelo Rasch (0,92 a 1,19), ocorrendo o mesmo com os índices de Outfit $(0,95$ a 1,26). A primeira dimensão foi denominada 
Clima e Funcionamento Organizacional (16 itens), e diz respeito ao ambiente físico inadequado, dificuldades pessoais com o superior, perspectiva profissional, salário inadequado para a função, dentre outros. A dimensão Pressão no Trabalho (13 itens) versa sobre acúmulo de funções, trabalhos que não pertencem à função, trabalho do outro, prazos para realização de trabalhos, ritmo acelerado, entre outros. A terceira dimensão (11 itens) denominou-se de Infraestrutura e Rotina, com itens referentes a equipamentos precários, licença de saúde recorrente dos colegas, mudança nas horas de trabalho, cooperação da equipe, entre outros. Os estudos de Rasch indicaram precisão de 0,98 para os itens e de 0,91 para as pessoas.

Beck Anxiety Inventory (BAI) (Cunha, 2001): constitui-se de 21 itens, e o indivíduo deve apontar, em uma escala de quatro pontos, o nível de gravidade do sintoma. O instrumento demonstrou-se apropriado para utilização não somente em pacientes psiquiátricos (sujeitos com transtornos de humor/ansiedade, dependência de álcool ou outras substâncias e quaisquer diagnósticos psiquiátricos com ideação ou tentativa suicida), mas também para a clínica médica (obesos, pessoas com doenças pulmonares ou cardíacas, disfunção erétil, unidade de cuidados primários) e para a população não clínica (universitários, bombeiros, adolescentes, idosos, funcionários de hospital e de outras empresas) (Cunha, 2001). O escore total varia de 0 a 63, e permite a verificação do nível de intensidade da ansiedade. A classificação descrita no manual recomenda que o nível de ansiedade seja classificado em mínimo (0-7), leve (8-15), moderado (16-25) ou grave (26-63).

No que se refere à validade do BAl, citam-se, a seguir, dois estudos importantes. O primeiro teve como objetivo buscar associação com o BDI, cujas correlações variaram de $r=0,48$ a $r=0,78$, em amostra psiquiátrica; de $r=0,25$ a $r=0,62$, em amostra médica, e $r=0,45$ a $r=0,76$, em amostra não clínica. Já no estudo de validação fatorial com a versão em português do BAl, participaram 379 pacientes com transtornos de ansiedade. A análise fatorial exploratória com rotação varimaxfoi utilizada, e obteve-se solução fatorial capaz de explicar 57,79\% da variância total, tendo o instrumento apresentado índices de precisão por alfa de Cronbach entre 0,83 e 0,92, em amostras não clínicas (Cunha, 2001).

\section{Procedimentos}

Após encaminhamento e aprovação do projeto ao comitê de ética (CAAE 0203.0.142.000-07), e autorizada a coleta de dados pelos coordenadores e docentes dos cursos, os instrumentos foram aplicados coletivamente em salas de aula com aproximadamente 30 alunos, mediante assinatura do Termo de Consentimento Livre e Esclarecido. A aplicação durou em torno de 40 minutos, sendo a ordem dos instrumentos mantida - BAI, EDEP e EVENT -, a fim de se intercalarem os instrumentos de construtos mais próximos, ou seja, a BAI e a EVENT.

\section{Resultados}

Primeiramente, foi realizada uma análise descritiva dos instrumentos para verificar o perfil da amostra estudada (Tabela 1).

A média de pontuação na BAl foi de 10 pontos, indicando que a amostra tem níveis mínimos de ansiedade. Quanto a EVENT, os escores no Fator Clima e Funcionamento Organizacional demonstraram que os participantes estão abaixo da média normativa desse grupo. O mesmo não ocorreu no Fator Pressão no Trabalho, em que a pontuação ficou acima da média do manual. Em relação ao terceiro fator, a pontuação encontra-se também abaixo da média. Por fim, a EVENT apresentou-se, em seu escore total, abaixo da média normativa e do ponto médio do instrumento.

Para a análise inferencial dos dados, realizou-se a estatística paramétrica, utilizando a correlação de Pearson para verificação das correlações entre os testes. O nível de significância adotado para as análises foi $p \leq 0,05$ (Tabela 2).

Tabela 1. Análise descritiva das médias, desvio-padrão e pontuação mínima e máxima dos três instrumentos.

\begin{tabular}{lcccc}
\hline & Mínima & Máxima & Média & DP \\
\hline EDEP (0-300) & 5 & 248 & 88,37 & 47,00 \\
BAI (0-63) & 0 & 54 & 10,00 & 9,33 \\
EVENT (0-80) & 0 & 61 & 30,38 & 14,55 \\
EVENT F1 (0-32) & 0 & 26 & 11,43 & 7,00 \\
EVENT F2 (0-26) & 0 & 25 & 13,78 & 6,22 \\
EVENT F3 (0-22) & 0 & 17 & 5,18 & 3,56 \\
\hline
\end{tabular}

DP: desvio-padrão; EDEP: escala de depressão; EVENT: escala de vulnerabilidade ao stress no trabalho; BAI: Beck anxiety inventory. 
Tabela 2. Correlação de Pearson entre a Escala de Depressão, o Inventário de Ansiedade de Beck e a Escala de Vulnerabilidade ao Stress Laboral.

\begin{tabular}{lcccccc}
\hline & & BAI Total & EVENT Clima & EVENT Pressão & EVENT Infraestrutura & EVENT Total \\
\hline EDEP & $r$ & 0,56 & 0,18 & 0,16 & 0,07 & 0,17 \\
Total & $p$ & 0,00 & 0,05 & 0,08 & 0,42 & 0,06 \\
BAI & $r$ & & 0,16 & 0,16 & 0,02 & 0,15 \\
Total & $p$ & - & 0,09 & 0,08 & 0,81 & 0,11 \\
\hline
\end{tabular}

EDEP: escala de depressão; EVENT: escala de vulnerabilidade ao stress no trabalho; BAI: Beck anxiety inventory.

Foram encontradas correlações positivas entre EDEP, BAl e EVENT. De acordo com a classificação estatística sobre magnitude de correlação, proposta por Sisto (2007), as correlações encontradas entre EVENT e EDEP foram baixas; no Fator Infraestrutura, foi o resultado mais próximo de zero, o que indica ausência de relação dos construtos. Na EDEP com BAl, a correlação foi moderada. Em relação à BAI com EVENT, os resultados não foram significativos, sendo as magnitudes fracas, com exceção da correlação com o fator 3 da EVENT, que foi nula.

\section{Discussão}

O presente estudo visou encontrar evidências de validade baseada na relação com outras variáveis para o instrumento inicialmente denominado Escala de Depressão (EDEP), mediante a utilização de outros instrumentos (BAl e EVENT). Como apontam as diretrizes da American Educational Research Association, the American Psychological Association, and the National Council on Measurement in Education (1999), as correlações são consideradas parâmetros importantes para se avaliarem evidências de validade baseada na relação com outras variáveis, já que dois instrumentos que meçam o mesmo construto devem apresentar correlações altas (convergência), enquanto instrumentos que meçam construtos relacionados podem apresentar correlações mais baixas, dependendo de quão relacionados são os construtos.

Nesse sentido, a literatura vem apontando correlações entre medidas de depressão e ansiedade com magnitudes que variam entre 0,40 e 0,70, independentemente de serem utilizadas amostras clínicas ou da população geral. Essa variação de magnitude decorre, 350 inicialmente, de modelos explicativos tripartidos de ansiedade e depressão, em que a sobreposição dos construtos estaria relacionada a três partes específicas. Uma primeira parte dos itens, tanto de escalas de depressão quanto de ansiedade, estaria relacionada a sintomas autonômicos, típicos da ansiedade. Uma segunda parte dos sintomas estaria relacionada à ausência de afeto positivo, típico da depressão; e uma parte dos sintomas seria comum aos dois construtos, especificamente o conjunto de sintomas relacionado ao afeto negativo (desconforto, insatisfação, medo, tristeza, desgosto, preocupação), que seriam os sintomas inespecíficos, presentes tanto na depressão quanto na ansiedade (Clark \& Watson, 1990; Otálvaro, 2007).

Watson et al. (1995) apontam que a avaliação clínica também demonstra esse modelo explicativo, além de haver grande dificuldade em encontrar pacientes "puros", ou seja, somente com sintomas de depressão ou somente com sintomas de ansiedade. Tal como descrito anteriormente, Beck et al. (1982) relatam que a ansiedade constitui-se um transtorno frequentemente apresentado pelos pacientes depressivos, principalmente quando eles experimentam uma queda da depressão. Nesse sentido, Watson et al. (1995) postulam que a correlação entre sintomatologia de depressão e ansiedade seria maior em casos de menor gravidade, enquanto em casos mais graves, a magnitude da correlação seria menor, já que haveria uma tendência em se encontrar a predominância dos fatores específicos de cada construto.

A magnitude correlacional obtida da relação entre EDEP e BAl foi moderada e positiva, indicando que quanto maior a ansiedade dos participantes, maiores os sintomas depressivos. Outros estudos, como os de Otálvaro (2007) e de Cunha (2001), vêm apontando correlações muito próximas das aqui encontradas entre os instrumentos que avaliam sintomatologia depressiva 
e de ansiedade, independentemente dos instrumentos utilizados.

Por fim, verificou-se que a correlação entre EVENT e seus fatores e EDEP foi classificada como fraca, e nula no fator 3 da EVENT. Tal resultado vai de encontro ao estudo de Grote et al. (2007), que verificaram relações mais consistentes entre os construtos, indicando que aqueles que apresentavam estressores crônicos com maior intensidade apareciam como potenciais para desenvolvimento de sintomas depressivos, e com o estudo de Isyanov e Calamari (2004), em que a percepção do stress apareceu como mediadora para a depressão. Resultados parcialmente convergentes a este estudo também foram encontrados por Palmeira (2009) e Oswaldo (2009), que, ao aplicaram as mesmas escalas (EDEP e EVENT) com amostras diferentes, encontraram correlações de maior magnitude entre depressão e vulnerabilidade ao stress no trabalho, apontando que a forma com que a avaliação do ambiente de trabalho é feita pode estar associada diretamente à saúde mental do sujeito, especificamente aos sintomas de depressão, assim como o afirmado por Lazarus (1976), Beck et al. (1982) e Pearlin (1982).

Por fim, é importante levar em consideração que o instrumento escolhido para avaliar o stress laboral mensura apenas eventos ambientais estressores e não sintomas de ansiedade; considera-se, assim, que tais resultados podem ser avaliados como coerentes.

\section{Considerações Finais}

O presente estudo teve um caráter exploratório para avaliar a validade da EDEP relacionada a outras variáveis, no caso, o stress laboral e a ansiedade. Conforme verificado, apesar de os estudos demonstrarem a relação de stress laboral com depressão, no presente estudo, isso não foi verificado de forma acentuada. No que se refere às relações da EDEP com BAl, nota-se que foi encontrada uma correlação de magnitude bastante adequada em relação à literatura científica, que vai ao encontro de estudos que demonstram a estreita ligação entre esses dois construtos.

Finalmente, consideram-se como limitações o tamanho amostral, a pouca variabilidade das medidas de stress laboral e de ansiedade desta amostra e o fato de a coleta de dados ter ocorrido em apenas um local e somente com universitários. Assim, sugere-se que outros estudos sejam realizados, levando-se em conta tais pontos, e que também sejam realizadas análises estatísticas que captem os principais indicadores da EDEP que se correlacionam com os de ansiedade, assim como a avaliação dessa relação mediante a aplicação em outras populações e com outros instrumentos validados para a depressão, de forma a comparar as correlações obtidas pela EDEP. Por fim, parece que a EDEP vem apresentando evidências de validade que sugerem a continuidade de seus estudos psicométricos.

\section{Referências}

American Educational Research Association. (1999). Standards for educational and psychological testing. Washington, DC: American Educational Research Association.

American Psychological Association. (2002). Manual diagnóstico e estatístico de transtornos mentais: DSM IV TR (4a ed.). Porto Alegre: Artmed.

Anxiety Disorders Association of America. (2009). Brief view of anxiety disorders. Retrieved on January 4, 2009, available from: <http://www.adaa.org/gettinghelp/ briefoverview.asp $>$.

Baptista, M. N. (2009). Escala de depressão. Relatório Técnico não-publicado. Programa de Pós Graduação Stricto-Sensu, Universidade São Francisco, Itatiba.

Beck, T. A., Rush, J. A., Shaw, F. B., \& Emery, G. (1982). Terapia cognitiva da depressão. Rio de Janeiro: Zahar.

Clark, L. A., \& Watson, D. (1990). Tripartide model of anxiety and depression: psychometric evidence and taxonomic implications. Journal of Abnormal Psychology, 100, 316-336.

Cordás, T. A. (1997). Do mal humorado ao mau humor: uma perspectiva histórica. In T. A. Cordás, A. E. Nardi, P. A. Moreno \& S. Castel. Distimia: do mau humor ao mal humor: diagnóstico e tratamento. Porto Alegre: Artes Médicas.

Corrêa, R. Z. A. (2008). Estresse laboral e qualidade de vida em enfermeiras: evidências de validade. Dissertação de mestrado não-publicada, Curso de Mestrado em Psicologia, Universidade São Francisco, Itatiba.

Crombach, L. (1996). Fundamentos de testagem psicológica (5 ed.). Porto Alegre: Artes Médicas.

Cunha, J. A. (2001). Manual da versão em português das escalas Beck. São Paulo: Casa do Psicólogo.

Fester, C. B. (1983). Functional analysis of depression. American Psychologist, 23 (10), 857-870.

Grote, N. K., Bledsoe, S. E., Larkin, J., Lemay, E. P., \& Brown, C. (2007). Stress exposure and depression in disadvantaged women: the protective effects of optimism and perceived control. Social Work Research, 31 (1), 19-33. 
Guic, E., Bilbao, M. A., \& Bertin, C. (2002). Estrés laboral y salud en uma muestra de ejecutivos chilenos. Revista Médica de Chile, 130 (10), 1101-1112.

Isyanov, E. V., \& Calamari, J. E. (2004). Does stress perception mediate the relationship between anxiety sensivity and anxiety and depression symptoms? Anxiety, Stress \& Cooping, 17 (2), 153-162.

Keenan, K., Feng, X., Hipwell, A., \& Klostermann, S. (2009). Depression begets depression: comparing the predictive utility of depression and anxiety symptoms of later depression. Journal of Child Psychology and Psychriatry, 50 (9), 1167-1175.

Lazarus, R. S. (1976). Patterns of adjustments. New York: MacGraw-Hill.

Mead, M. (s.d.). Teorias da etiologia da depressão. Recuperado em novembro 20, 2010, disponível em: <http://langpair=en|pt\&u=http://priory.com/homol/ dephist.htm>

Morais, P. R., Baptista, M. N., Calais, S. L., \& Inocente, N. J. (2004). Psicobiologia do estresse e da síndrome de burnout. In M. Z. Brandão, F. C. S. Conte, F. S. Brandão, V. K. Ingberman. V. L. M. Silva \& S. M. Oliani (Orgs.), Sobre comportamento e cognição: entendendo a psicologia comportamental e cognitiva aos contextos da saúde, das organizações, das relações pais efilhos e das escolas (Vol. 14, pp.96-110). São Paulo: ARBytes.

Organização Mundial da Saúde. (1993). Classificação internacional de doenças - CID10. Porto Alegre: Artes Médicas.

Oswaldo, Y. C. (2009). Vulnerabilidade ao estresse no trabalho, coping, depressão e qualidade devida: evidências devalidade. Tese de doutorado não-publicada, Programa de Pós- Graduação Stricto Sensu em Psicologia, Universidade São Francisco, Itatiba.

Otálvaro, L. E. O. (2007). Análisis correlacional del cuestionario de depressión estado-rasgo com uma muestra de adolescentes y universitários de la ciudad de melellín (Colombia). Psicología desde el Caribe, 20, 28-49.

Palmeira, P. P. G (2009). Depressão, estresse no trabalho e coping em policiais: estudo de evidência de validade-EDEP. Dissertação de mestrado não-publicada, Programa de Pós-Graduação Stricto Sensu em Psicologia, Universidade São Francisco, Itatiba.

Pasquali, L. (1999). Instrumentos psicológicos: manual prático de elaboração. Brasília: LabPAM.

Pearlin, L. I. (1982). The social contexts of stress. In L. Goldberger \& S. Breznit (Orgs.). Handbook of stress: theorical and clinical aspects. London: Collier MacMillan Publishers.
Pereira, D. A. P., \& Amaral, V. L. A. (2004). Escala de avaliação de depressão para crianças: um estudo de validação. Estudos em Psicologia (Campinas), 21 (1), 5-23. doi: 10.15 90/S0103-166X2004000100001.

Sadock, B. I., \& Sadok, V. A. (2007). Compêndio de psiquiatria: ciência do comportamento e psiquiatria clínica (9a ed.). Porto Alegre: Artmed.

Schnittker, J. (2010). Gene environment correlations in the stress-depression relationship. Journal of Health and Social Behavior, 51 (3), 229-243.

Sisto, F. F. (2007). Delineamento correlacional. In M. N. Baptista \& D. C. Campos, Metodologias de pesquisa em ciências: análise quantitativa e qualitativa (pp. 90-101). Rio de Janeiro: LTC.

Sisto, F. F., Baptista, M. N., Noronha, A. P., \& Santos, A. A. A. (2007). Escala de vulnerabilidade ao estresse no trabalho. São Paulo: Vetor.

Souza, M. S. (2010). Evidências de Validade e Precisão para a Escala de Depressão de Baptista e Sisto (EDEP). Tese de doutorado não-publicada, Programa de Pós-Gra-duação Stricto Sensu em Psicologia, Universidade São Francisco, Itatiba.

Sparrenberger, F., Santos, I., \& Lima, R. C. (2004). Associação de eventos de vida produtores de estresse e mal-estar psicológico: um estudo de base populacional. Cadernos de Saúde Pública, 20 (1), 249-258.

Urbina, S. (2007). Fundamentos da testagem psicológica. Porto Alegre: Artmed.

Warner, V., Wickramaratne, P., \& Weissman, M. M. (2008). The role of fear and anxiety in the familial risk for major depression: a three generation study. Psychological Medicine, 38, 1543-1556.

Watson, D., Clark, L. A., Weber, K., Assenheimer, J. S., Strauss, M. E., \& Mccormick, R. A. (1995). Testing a tripartite model: II. Exploring the symptom structure of anxiety and depression in student, adult, and patient samples. Journal of Abnormal Psychology, 104 (1), 15-25.

World Health Organization. (2006). Conquering depression: mental health and substance abuse. Retrieved April 10, 2008, available from: <http://www.searo.who.int>.

Wong, M. (2007). História e epidemiologia da depressão. In J. Licinio \& M. Wong. Biologia da depressão (pp.15-25). Porto Alegre: Artmed.

Recebido em: 16/9/2009

Versão final reapresentada em: 2/12/2010

Aprovado em: 4/2/2011 